\title{
CT Features of COVID-19: A Case Report
}

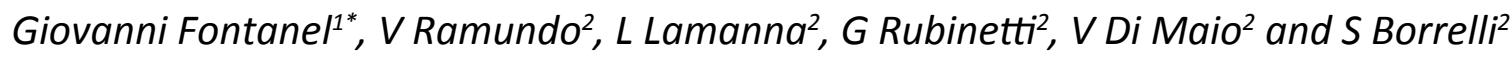 \\ ${ }^{1}$ Department of Radiology, Ospedale Sacro Cuore di Gesù-Fatebenefratelli, Italy \\ ${ }^{2}$ Centro Diagnostico "Alta Irpinia", Italy
}

\begin{abstract}
We describe here the Chest $\mathrm{CT}$ imaging features of a 68-year-old man presenting with fever $\left(37.5^{\circ} \mathrm{C}\right)$, malaise, dyspnea and persistent dry cough for 7 days. The patient was seen by his GP, then sent to us for a CT and treated as a suspect even before exam execution, with all PPE and safety measures adequately put in place. Chest CT showed strikingly remarkable signs of interstitial pneumonia, compatible with COVID-19. The patient was immediately subsequently referred to the local COVID center, where the suspected diagnosis was confirmed.
\end{abstract}

\section{Discussion}

The exam was executed following the indications sent in mid-March 2020 by the Italian Society of Radiology (SIRM) [1]: Supine, volumetric, thin-slice (1 mm), non-contrast and high-resolution acquisition, in inspiration, visualized with correct windowing (W/W 1500/-600).

As pointed out in this [2] case collection from patients in Wuhan, China, it appears we scanned our patient while in the second week from the start of the disease, the one in which focal, unilateral ground-glass opacities progress to diffuse bilaterally and rather symmetrically.

In fact, the pattern we found in our patient strikingly resembles that of patients examined in Rome in this prospective study [3]: The images show bilateral, posterior,

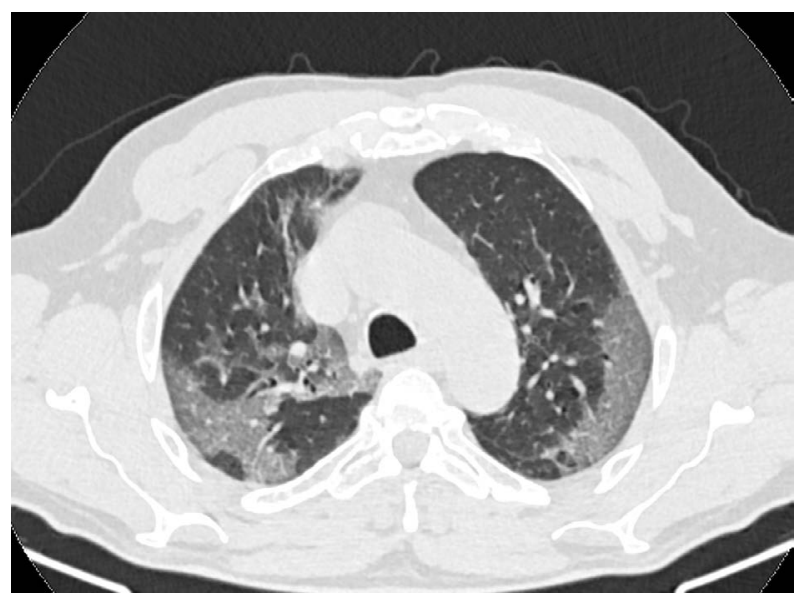

Figure 1: Transverse section, thin-slice $(1 \mathrm{~mm})$ : Bilateral, posterior, peripheral diffuse ground-glass opacities, with illdefined margins and multi lobe involvement. peripheral diffuse ground-glass opacities, with ill-defined margins and multi-lobe involvement (Figure 1).

While all lobes in both lungs are affected, there is a

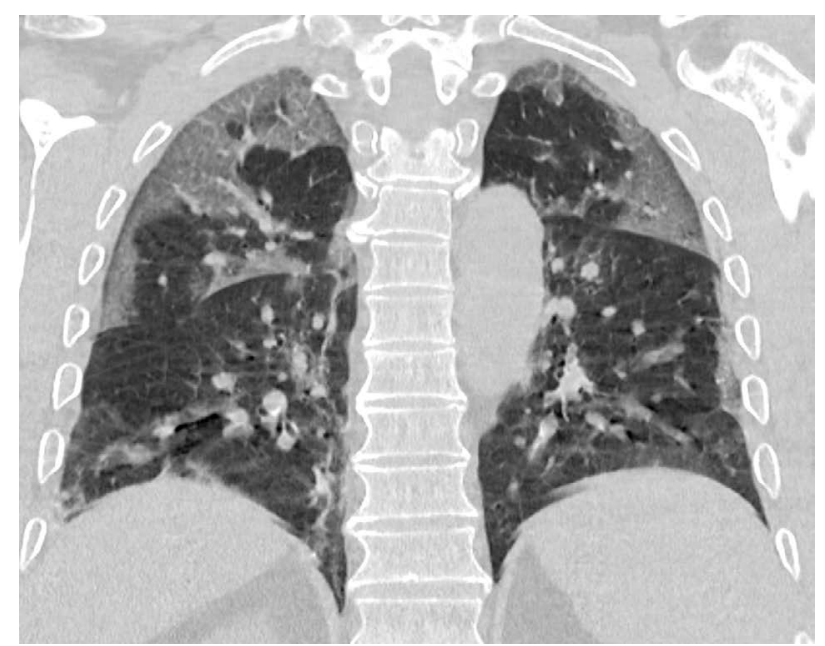

Figure 2: Coronal posterior section. Bilateral, peripheral, almost symmetrical ground-glass opacities mainly affecting the Upper Lung lobes and upper segments of both lower lung lobes.

*Corresponding author: Giovanni Fontanel, Department of Radiology, Ospedale Sacro Cuore di Gesù-Fatebenefratelli, Benevento, Italy

Received: April 15, 2020

Accepted: April 27, 2020

Published online: April 29, 2020

Citation: Fontanel G, Ramundo V, Lamanna L, et al. (2020) CT Features of COVID-19: A Case Report. Sch J Emerg Med Crit Care $4(1): 71-72$

Copyright: (c) 2020 Fontanel G, et al. This is an open-access article distributed under the terms of the Creative Commons Attribution License, which permits unrestricted use, distribution, and reproduction in any medium, provided the original author and source are credited. 


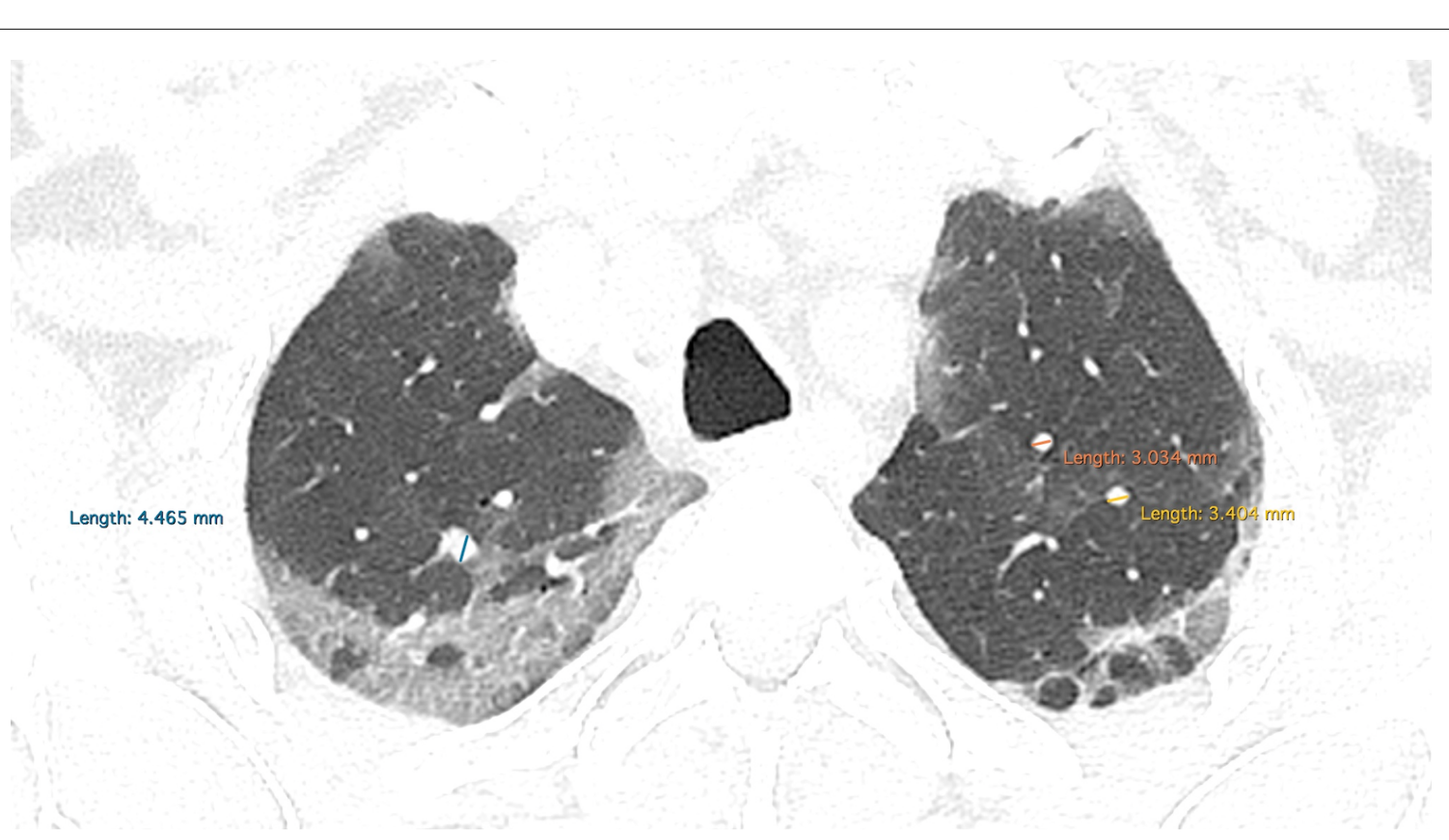

Figure 3: Transverse section at the Upper Lung Lobes. Sub-segmental vessel enlargement in both upper lung lobes ( $\varnothing \mathrm{max} 4.4 \mathrm{~mm}$, in the Right Upper Lung Lobe).

clear predominance for upper lobes and the upper segment of both lower long lobes (Figure 2).

Slightly enlarged sub segmental vessels (Figure 3) [3], especially in the upper lung lobes was noted ( $\varnothing$ 3-4.4 $\mathrm{mm}$ ), a finding first reported by Albarello, et al. [4] and Bai, et al. [5]. $\mathrm{Ye}$, et al. [6] noted the finding as well, linking it to pro-inflammatory factors, one of them being IL-6, and the Cytokine Release Syndrome, responsible for the more severe and often fatal COVID; this often overlooked finding might be then of high importance in assessing patient susceptibility to Tocilizumab, an IL-6 receptor antagonist, which has shown to drastically reduce mortality in severe COVID patients [6].

No consolidations, signs of a later-stage disease, at least in week three [2], were reported. No air-bronchogram, nodules, pleural effusion or lymphadenopathy were observed.

\section{Conclusion}

Our patient showcases the typical chest CT COVID-19 findings: Bilateral, posterior, peripheral diffuse ground-glass opacities, with ill-defined margins and multi-lobe involvement.
The sub-segmental vessel enlargement we noted, already widely reported in literature, should not be overlooked and reported, as it could be a CT marker for more severe cases susceptible to antibody therapy.

\section{References}

1. https://www.sirm.org/wp-content/uploads/2020/03/Tecnicheesecuzione-HRCT-in-pazienti-COVID-19.pdf

2. Shi $H$, Han $X$, Jiang $N$, et al. (2020) Radiological findings from 81 patients with COVID-19 pneumonia in Wuhan, China: A descriptive study. Lancet Infect Dis 20: 425-434.

3. Damiano Caruso, Marta Zerunian, Michela Polici, et al. (2020) Chest CT Features of COVID-19 in Rome, Italy. Radiology.

4. Albarello F, Pianura E, Di Stefano F, et al. (2020) 2019-novel Coronavirus severe adult respiratory distress syndrome in two cases in Italy: An uncommon radiological presentation. Int J Infect Dis 93: 192-197.

5. Bai HX, Hsieh B, Xiong Z, et al. (2020) Performance of radiologists in differentiating COVID-19 from viral pneumonia on chest CT. Radiology.

6. Ye Z, Zhang $Y$, Wang $Y$, et al. (2020) Chest CT manifestations of new coronavirus disease 2019 (COVID-19): A pictorial review. Eur Radiol.

DOI: $10.36959 / 592 / 384$ 